\title{
Microarray-based DNA methylation study of Ewing's sarcoma of the bone
}

\author{
HYE-RIM PARK ${ }^{1}$, WOON-WON JUNG ${ }^{2}$, HYUN-SOOK KIM ${ }^{3}$ and YONG-KOO PARK ${ }^{4}$
}

\author{
${ }^{1}$ Department of Pathology, College of Medicine, Hallym University, Anyang, Gyeonggi 431-070; \\ ${ }^{2}$ Department of Biomedical Laboratory Science, College of Health Science, Cheongju University, Cheongju, \\ Chungbuk 360-764; ${ }^{3}$ Department of Biomedical Laboratory Science, College of Health Science, Korea University, \\ Seoul 136-703; ${ }^{4}$ Department of Pathology, College of Medicine, Kyung Hee University, Seoul 130-702, Republic of Korea
}

Received October 4, 2013; Accepted May 13, 2014

DOI: $10.3892 / 01.2014 .2322$

\begin{abstract}
Alterations in DNA methylation patterns are a hallmark of malignancy. However, the majority of epigenetic studies of Ewing's sarcoma have focused on the analysis of only a few candidate genes. Comprehensive studies are thus lacking and are required. The aim of the present study was to identify novel methylation markers in Ewing's sarcoma using microarray analysis. The current study reports the microarray-based DNA methylation study of $1,505 \mathrm{CpG}$ sites of 807 cancer-related genes from 69 Ewing's sarcoma samples. The Illumina GoldenGate Methylation Cancer Panel I microarray was used, and with the appropriate controls $(n=14)$, a total of 92 hypermethylated genes were identified in the Ewing's sarcoma samples. The majority of the hypermethylated genes were associated with cell adhesion, cell regulation, development and signal transduction. The overall methylation mean values were compared between patients who survived and those that did not. The overall methylation mean was significantly higher in the patients who did not survive $(0.25 \pm 0.03)$ than in those who did $(0.22 \pm 0.05)(\mathrm{P}=0.0322)$. However, the overall methylation mean was not found to significantly correlate with age, gender or tumor location. GDF10, OSM, APC and HOXA11 were the most significant differentially-methylated genes, however, their methylation levels were not found to significantly correlate with the survival rate. The DNA methylation profile of Ewing's sarcoma was characterized and 92 genes that were significantly hypermethylated were detected. A trend towards a more aggressive behavior was identified in the methylated group. The results of this study indicated that methylation may be significant in the development of Ewing's sarcoma.
\end{abstract}

Correspondence to: Professor Yong-Koo Park, Department of Pathology, College of Medicine, Kyung Hee University, 1 Hoeki-dong, Dongdaemun-ku, Seoul 130-702, Republic of Korea E-mail: ykpark@khmc.or.kr

Key words: microarray, DNA methylation, Ewing's sarcoma

\section{Introduction}

Ewing's sarcoma is the second most common type of solid bone and soft-tissue malignancy in children and young adults, and has low cure rates, indicating the requirement to identify further prognostic markers. It has been widely accepted that the acquisition of genetic changes is essential in the development of malignancies. Such alterations include irreversible changes in the DNA sequence, including mutations, translocations, deletions and amplifications, which result in gene activation or inactivation. Epigenetic changes, which represent reversible modifications that affect gene expression without altering the DNA sequence itself, are also a hallmark of cancer (1).

The field of epigenetics describes information transmission through the cell division of heritable changes in a phenotype that does not involve DNA sequence changes and is transferred by cell division. $\mathrm{CpG}$ island hypermethylation, histone modification and transmitted chromatin structures are mechanisms underlying epigenetic transmission, and among these, $\mathrm{CpG}$ island hypermethylation is a key component for altered gene expression associated with human cancer (2). Although the causes are unclear, promoter $\mathrm{CpG}$ island hypermethylation may be associated with aging and cancer development (2). Promoter $\mathrm{CpG}$ island hypermethylation is found in virtually all human cancer tissue types and acts as an important mechanism for the inactivation of tumor suppressor and tumor-related genes (3).

Aberrant DNA methylation has been recognized as an early event in tumorigenesis $(4,5)$ and therefore, variations in the methylation patterns identified between normal and tumor cells may aid in the detection of tumor cells in biopsy specimens or tumor-derived DNA in body fluids (6). The advent of high-throughput microarray technology allows for the simultaneous evaluation of genome-wide DNA methylation patterns and RNA expression levels in tumor specimens, and also allows for the identification of molecular targets or gene classifiers that are specific to tumor cells (7).

The number of genes demonstrated to be inactivated by promoter $\mathrm{CpG}$ island hypermethylation has abruptly increased with the application of array-based genome-scale DNA methylation analysis. The GoldenGate assay for methylation has 
successfully analyzed the methylation profiles of $1,536 \mathrm{CpG}$ sites from 371 genes identified in cancer cell lines, lung cancer and normal tissues, and has identified a panel of markers specific for adenocarcinoma methylation $(8,9)$. The assay has also been used to assess the epigenetic specificity of the loss of IGF2 imprinting in Wilms' tumors and to identify the unique epigenetic signature of human embryonic stem cells $(10,11)$. However, there is little information concerning similar broad-based studies of Ewing's sarcoma. The objective of the present study was to analyze methylation patterns and to assess their clinical significance in Ewing's sarcomas.

\section{Materials and methods}

Ewing's sarcoma samples and controls. This study was approved by the Institutional Review Board of Kyung Hee University Hospital (Seoul, Korea). A total of 69 samples from patients with Ewing's sarcomas were analyzed. The disease was diagnosed based on the World Health Organization criteria (12). Briefly, Ewing's sarcoma is a small round cell sarcoma, with diffuse membranous CD99 immunostaining, cytoplasmic periodic acid-Schiff staining and EWSRl gene translocation, as determined using Zytolight SPEC ROSI and RET Dual color break apart probes and demonstrated with fluorescence in situ hybridization, according to the manufacturer's instructions (Zytovision, Bremerhaven, Germany). The clinical features of the patients are summarized in Table I. The age at diagnosis ranged between one and 57 years, and 39 patients were male and 30 were female. The primary sites were the long bones $(n=31)$ and the flat, small bones and spine $(\mathrm{n}=38)$. Metastasis at diagnosis was present in five patients. The follow-up data were only available for 37 patients and the follow-up durations ranged between six and 240 months. As control samples, 14 tissue specimens were used that were obtained from the cancellous bone during total hip or knee joint replacement surgeries due to degenerative osteoarthritis. These included the marrow components of the tibia or femur.

Preparation of DNA samples. DNA extraction was performed as described previously (13). Genomic DNA was extracted from the formalin-fixed, paraffin-embedded (FFPE) tissue sections of each sample by a Magna Pure LC instrument (Roche Diagnostics GmbH, Mannheim, Germany). Briefly, $10-\mu \mathrm{m}$ paraffin sections were mixed gently with $800 \mu \mathrm{l}$ xylol and $400 \mu \mathrm{l}$ absolute ethyl alcohol by inverting the tube several times. The supernatant was discarded following brief centrifugation at $12,000 \mathrm{x}$ g for $10 \mathrm{mins}$, and the pellet was washed with $1 \mathrm{ml}$ absolute ethyl alcohol. The pellet was dried for $10 \mathrm{~min}$ at $55^{\circ} \mathrm{C}$ following removal of the supernatant. The tissue pellet was vortexed with $80 \mu \mathrm{l}$ of a tissue lysis buffer (Roche diagnostics $\mathrm{GmbH}$ ) and $20 \mu \mathrm{l}$ proteinase $\mathrm{K}$, followed by overnight incubation at $55^{\circ} \mathrm{C}$. The digested samples were loaded into the Magna Pure LC instrument. All DNA samples were stored at $-70^{\circ} \mathrm{C}$ prior to use. The DNA concentrations derived from the FFPE samples were determined on a fluorophotometer (Victor3; Perkin-Elmer, Waltham, MA, USA), using the PicoGreen nucleic acid quantification kit (PicoGreen; Molecular Probes, Eugene, OR, USA), which allows accurate and reproducible DNA quantification at low concentrations, including DNA extracted from archival FFPE samples (14).
Table I. Overall methylation mean values of Ewing's sarcoma samples according to the clinical parameters.

\begin{tabular}{lcc}
\hline Parameter & $\begin{array}{c}\text { Overall } \\
\text { methylation mean }\end{array}$ & P-value \\
\hline Age, years & & 0.3659 \\
$\quad<20(\mathrm{n}=46)$ & $0.23 \pm 0.04$ & \\
$\geq 20(\mathrm{n}=23)$ & $0.24 \pm 0.04$ & \\
Gender & & 0.3304 \\
$\quad$ Male $(\mathrm{n}=39)$ & $0.24 \pm 0.04$ & \\
Female ( $\mathrm{n}=30)$ & $0.23 \pm 0.04$ & \\
Location & & 0.0761 \\
$\quad$ Long bone (n=31) & $0.23 \pm 0.04$ & \\
Flat, small bone, spine $(\mathrm{n}=38)$ & $0.24 \pm 0.04$ & \\
Survival & & $0.0322^{\mathrm{a}}$ \\
$\quad$ Not alive $(\mathrm{n}=18)$ & $0.25 \pm 0.03$ & \\
Alive (n=19) & $0.22 \pm 0.05$ & \\
\hline
\end{tabular}

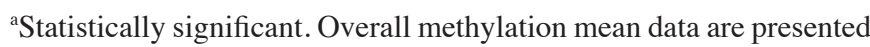
as the mean \pm standard deviation.

Bisulfite conversion and methylation chip assay. Bisulfite conversions of all DNA samples were performed using an EZ-96 DNA methylation kit (Zymo Research Corporation, Orange, CA, USA) according to the manufacturer's instructions. In total, $500 \mathrm{ng}$ of genomic DNA was used for each bisulfite conversion. Following bisulfite treatment, the quantification of the methylcytosine content was performed using the Illumina GoldenGate Methylation Cancer Panel I microarray, as described previously (3). The GoldenGate Methylation Cancer Panel I product was used to process $1,505 \mathrm{CpG}$ sites from a panel of 807 cancer-related genes, which included oncogenes and genes associated with DNA repair, tumor suppression, cell cycle, differentiation and apoptosis. Of these 1,505 CpG sites, 1,044 were located within $\mathrm{CpG}$ islands and 461 were located outside $\mathrm{CpG}$ islands. Briefly, the bisulfite-converted DNA was reacted with biotin and hybridized to assay oligos. Next, specific extensions and ligations were performed at $45^{\circ} \mathrm{C}$ for $15 \mathrm{~min}$. The ligated products were amplified by polymerase chain reaction (PCR) and conditioned as follows: $10 \mathrm{~min}$ at $37^{\circ} \mathrm{C}$; then 34 cycles of $35 \mathrm{sec}$ at $95^{\circ} \mathrm{C}, 35 \mathrm{sec}$ at $56^{\circ} \mathrm{C}, 2 \mathrm{~min}$ at $72^{\circ} \mathrm{C} ; 10 \mathrm{~min}$ at $72^{\circ} \mathrm{C}$; and cooling for $5 \mathrm{~min}$ at $4^{\circ} \mathrm{C}$. Single-stranded PCR products were prepared by denaturation and hybridized to a Sentrix Array Matrix (GoldenGate Methylation Cancer Panel I). The array hybridization was conducted overnight under a temperature gradient program ranging from 45 to $60^{\circ} \mathrm{C}$, and arrays were imaged using a Bead Array Reader scanner (Illumina, San Diego, CA, USA). The raw methylation ratios were calculated using the Illumina BeadStudio Methylation Module (Illumina) following background normalization, which was derived by averaging the signals of the built-in negative control (15). Each sample was examined in a duplicate manner in the chip assay.

Statistical analysis. Data were analyzed using SAS version 4 (SAS Institute, Cary, NC, USA). The two-sample t-test and a 
Table II. List of methylated genes in Ewing's sarcoma.

\begin{tabular}{ll}
\hline Function & \\
\hline $\begin{array}{l}\text { Cell adhesion }(\mathrm{n}=10) \\
\text { Cell cycle }(\mathrm{n}=4)\end{array}$ & APC, GP1BB, LAMC1, CD2, THBS2, ITGB1, COL18A1, APBA1, CD34, BCAM \\
Cell regulation $(\mathrm{n}=22)$ & CDKN, KLK10, PCTK1, KRAS \\
& OSM, TNFSF8, ETS1, EFNB3, LTB4R, FGF9, NGFR, WNT1, CASP8, TAL1, MAPK14, \\
Development $(\mathrm{n}=13)$ & GLC1, KDR, CEACAM1, LYN, PTPN6, F2R, TNFSF10, PTHLH, CD86, CASP10, ESR1 \\
& ZP3, TBX1 \\
Immune response $(\mathrm{n}=4)$ & IL18BP, STAT5A, IRF7, HLA-DRA \\
Metabolism $(\mathrm{n}=4)$ & EYA4, LRP2, FVT1, GPX1 \\
Protein regulation $(\mathrm{n}=5)$ & DIO3, VAMP8, GNMT, CAPG, TJP2 \\
Signal transduction $(\mathrm{n}=25)$ & GABRB3, SLC22A3, LAT, EPHA1, GP1BB, EVI2A, CD81, IGF2R, KCNQ1, HCK, NTSR1, \\
& ITPR2, S100A4, SH3BP2, EPHA7, EPHA3, TES, NEFL, TMEFF2, MAP3K1, VAV1, NGFB, \\
& IRAK3, RHOH, STK11 \\
Transcription regulation $(\mathrm{n}=5)$ & HOXA11, SOX17, ETV1, JUNB, TERT \\
\hline
\end{tabular}

Table III. Methylation profiles of four unique genes in Ewing's sarcoma samples according to survival rate.

\begin{tabular}{lccc}
\hline Gene & Not alive $(n=18)$ & Alive $(n=19)$ & P-value \\
\hline GDF10 & $0.53 \pm 0.34$ & $0.52 \pm 0.35$ & 0.4528 \\
OSM & $0.46 \pm 0.38$ & $0.44 \pm 0.37$ & 0.4475 \\
APC & $0.51 \pm 0.35$ & $0.55 \pm 0.28$ & 0.3305 \\
HOXA11 & $0.29 \pm 0.37$ & $0.38 \pm 0.40$ & 0.2336 \\
\hline
\end{tabular}

Data are presented as the mean \pm standard deviation.

one-way analysis of variance were performed to estimate the methylation profiles on clinical parameters and survival rate. $\mathrm{P}<0.05$ was considered to indicate a statistically significant difference.

\section{Results}

The DNA methylation status of 69 Ewing's sarcoma samples was examined using the Illumina GoldenGate Methylation Cancer Panel I microarray. The GoldenGate DNA methylation assay measures the DNA methylation levels of a given locus as $\beta$-values ranging from 0 (no DNA methylation detected) to 1 (complete DNA methylation). The methylation patterns of the Ewing's sarcoma samples were extremely heterogeneous with respect to total DNA methylation. The criteria for differentially-methylated genes were applied to detect the genes whose DNA methylation levels $(\beta)$ differed by at least 0.15 between the Ewing's sarcoma and control samples (8). Therefore, a list of 92 differentially-methylated genes was obtained from the Ewing's sarcoma samples. The 92 genes were classified into nine groups based on their biological functions: i) Cell adhesion $(n=10)$; ii) cell cycles $(n=4)$; iii) cell regulation $(n=22)$; iv) development $(n=13)$; v) immune response $(n=4)$; vi) metabolism $(n=4)$; vii) protein regulation $(\mathrm{n}=5)$; viii) signal transduction $(\mathrm{n}=25)$; and ix) transcription regulation $(n=5)$. A list of the methylated genes identified in
Ewing's sarcoma is shown in Table II. Fig. 1 shows a heat map of the differentially-methylated CpGs in the Ewing's sarcoma samples.

The overall methylation mean of each tumor was compared according to survival status. The overall methylation mean was significantly higher in the patients who did not survive $(0.25 \pm 0.03)$ compared with the surviving patients $(0.22 \pm 0.05)$ $(\mathrm{P}=0.0322)$. However, no significant correlation was identified between the overall methylation mean and the clinical parameters of age, gender and tumor location (Table I).

Using a highly stringent selection criteria (a $\beta$ difference of <0.5), four unique genes (GDF, OSM, APC and HOXA11) were selected that were the most significantly differentially-methylated genes in the Ewing's sarcoma samples. The methylation of these top four genes was confirmed to be common, occurring in $82.5 \%(G D F), 65 \%(O S M), 87.5 \%$ $(A P C)$ and $45 \%$ (HOXA11) of the Ewing's sarcoma samples. However, their methylation levels were not found to significantly correlate with the survival rate (Table III).

\section{Discussion}

Ewing's sarcoma is comprised of morphologically heterogeneous tumors that are themselves characterized by non-random chromosomal translocations of the EWS gene and one of the members of the ETS family of transcription factors. The $(11 ; 22)(\mathrm{q} 24 ; \mathrm{q} 12)$ translocation is the most frequently occurring, and results in the formation of the EWS-FLI1 fusion protein. This protein aids Ewing's sarcoma pathogenesis by modulating target gene expression (16). However, only a few studies have analyzed gene methylation in Ewing's sarcoma. These studies have reported that the hypermethylation of $\mathrm{HICl}$, $M G M T, C D H 1, p 15$ and p16 in tumors, as well as the hypermethylation of CASPASE 8, occurs only in Ewing's sarcoma cell lines $(6,17-19)$. The number of genes that have been shown to be inactivated by promoter $\mathrm{CpG}$ island hypermethylation has abruptly increased with the application of array-based genome-scale DNA methylation analysis. However, there is little information concerning similar broad-based methylation studies on Ewing's sarcoma. The main goal of the present 


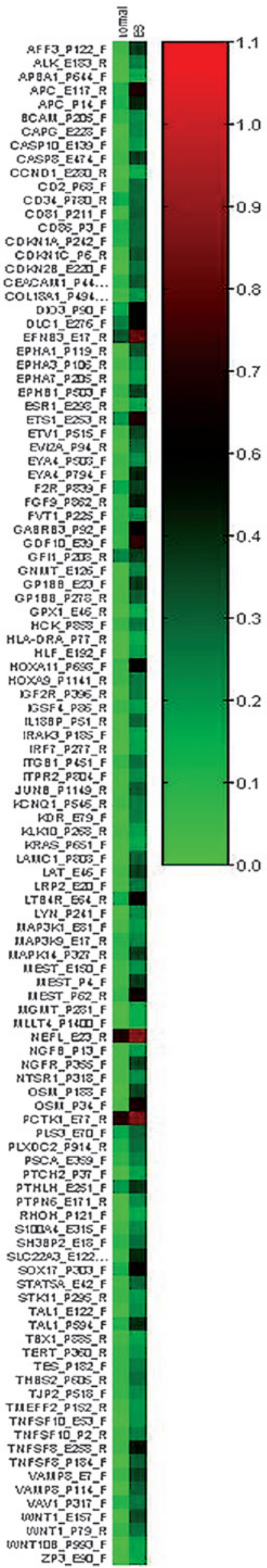

Figure 1. Heat map showing the differentially-methylated CpGs in the Ewing's sarcoma samples. Red, methylated genes; green, unmethylated genes. study was to provide a general overview of the changes in DNA methylation associated with Ewing's sarcoma.

The GoldenGate Cancer Panel I used in this study offers the ability to analyze 1,505 single $\mathrm{CpG}$ loci corresponding to 807 genes in parallel. The reproducibility and accuracy of this array-based approach have been extensively demonstrated $(15,20)$. Using the aforementioned criteria, 92 differentially-hypermethylated genes were identified in Ewing's sarcoma. These included numerous genes known to affect tumorigenesis by affecting cell regulation, signal transduction and differentiation. These results demonstrated that the GoldenGate assay offers a high-throughput method to identify novel genes with promoter DNA methylation.

Promoter $\mathrm{CpG}$ island hypermethylation can be used as a tumor biomarker that is able to detect tumor cells in serum or to predict clinical outcome. The simultaneous hypermethylation of multiple $\mathrm{CpG}$ island loci, particularly the $\mathrm{CpG}$ island methylator phenotype (CIMP), may be associated with survival rather than individual gene hypermethylation. The widespread hypermethylation of multiple promoter $\mathrm{CpG}$ island loci characterizes a subset of malignancies, designated as CIMP (2). The clinicopathological features of CIMP-positive Ewing's sarcoma remain obscure, and marker panels for diagnosing CIMP-positive Ewing's sarcoma have not yet been established.

The overall methylation mean of the tumor samples was compared with survival rate, and the overall methylation mean was significantly higher in the patients who did not survive compared with those who did. A trend towards a more aggressive behavior was identified in the methylated samples. However, no significant correlation was identified between the overall methylation mean and the clinical parameters of age, gender and tumor location. Thus, four unique genes (GDF10, $O S M, A P C$, and $H O X A 11)$ were selected that were the most significantly differentially methylated in the Ewing's sarcoma samples. The top four hypermethylated genes may function as differential epigenetic biomarkers between Ewing's sarcoma and control samples. However, their methylation levels were not found to significantly correlate with the survival rate.

Among these genes, GDF10 has been reported to have a gender-dependent effect on glioblastoma progression and survival (21). The location of the OSM gene has been found to be distal to the translocation breakpoint on chromosome 22 of Ewing's sarcoma (22). Higher levels of OSM have also been reported in metastasizing prostate cancer compared with non-metastasizing prostate cancer and benign prostatic hyperplasia (23). Furthermore, APC promoter hypermethylation has been shown to be an early event in endometrial tumorigenesis (24). HOX genes are important members of the homeobox superfamily, encoding transcription factors and contributing to oncogenesis through the activation of anti-apoptotic pathways (25). Fiegl et al (26) revealed that HOXA11 gene DNA methylation frequently occurs in ovarian cancer and that consequently, HOXA11 methylation status is a prognostic marker.

Avigad et al (27) analyzed the aberrant methylation of RASSF1A in Ewing's sarcoma using methylation-specific PCR. The study stated that Ewing's sarcoma patients with methylated RASSF1A showed poorer prognoses than those without. However, contrasting results were identified in the current study. In addition, Harada et al (28) reported that there 
is no methylation of RASSF1A in Ewing's sarcoma. These discordant results may be due to differences in the detection method or in the study population.

The results of the present study not only provide novel insights into the biology of Ewing's sarcoma, but also have potential therapeutic implications. DNA methylation inhibitors, such as decitabine and 5-azacitidine, are currently used in clinical studies to treat myelodysplastic syndrome or acute myeloid leukemia patients (29). If DNA methylation inhibitors exert therapeutic effects on the demethylation of hypermethylated genes, Ewing's sarcoma with a higher level of DNA hypermethylation may theoretically be a better target for such drugs. Thus, DNA methylation profiling may be a useful approach to monitoring the association between epigenetic and clinical responses, and to stratify patients for treatment with demethylating agents.

In conclusion, the current study identified the hypermethylation of 92 genes in Ewing's sarcoma and a trend toward more aggressive behaviors in samples with methylation. The top four hypermethylated genes can be used as differential epigenetic biomarkers for Ewing's sarcoma. However, further studies on their clinical implications with a larger scale sample are required to confirm these results.

\section{Acknowledgements}

The authors would like to thank Ye Won Bang and the Clinical Research Coordinating Center for the statistical analysis consultations.

\section{References}

1. Esteller M: Epigenetics in cancer. N Engl J Med 358: 1148-1159, 2008.

2. Kang GH: $\mathrm{CpG}$ island hypermethylation in gastric carcinoma and its premalignant lesions. Korean J Pathol 46: 1-9, 2012.

3. Esteller M, Com PG, Baylin SB and Herman JG: A gene hypermethylation profile of human cancer. Cancer Res 61: 3225-3229, 2001 .

4. Suzuki H, Watkins DN, Jair KW, et al: Epigenetic inactivation of SFRP genes allows constitutive WNT signaling in colorectal cancer. Nat Genet 36: 417-422, 2004.

5. Ushijima T: Detection and interpretation of altered methylation patterns in cancer cells. Nat Rev Cancer 5: 223-231, 2005.

6. Tsuchiya T, Sekine K, Hinohara S, Namiki T, Nobori T and Kaneko Y: Analysis of the p16INK4, p14ARF, p15, TP53, and MDM2 genes and their prognostic implications in osteosarcoma and Ewing sarcoma. Cancer Genet Cytogenet 120: 91-98, 2000.

7. Hoque MO, Kim MS, Ostrow KL, et al: Genome-wide promoter analysis uncovers portions of the cancer methylome. Cancer Res 68: 2661-2670, 2008.

8. Bibikova M, Lin Z, Zhou L, et al: High-throughput DNA methylation profiling using universal bead arrays. Genome Res 16: 383-393, 2006

9. Lin Z, Thomas NJ, Bibikova M, et al: DNA methylation markers of surfactant proteins in lung cancer. Int J Oncol 31: 181-191, 2007.
10. Bibikova M, Chudin E, Wu B, et al: Human embryonic stem cells have a unique epigenetic signature. Genome Res 16: 1075-1083, 2006.

11. Bjornsson HT, Brown LJ, Fallin MD, et al: Epigenetic specificity of loss of imprinting of the IGF2 gene in Wilms tumors. J Natl Cancer Inst 99: 1270-1273, 2007.

12. de Alvia E, Lessnick SL and Sorensen PH: Ewing sarcoma. In: WHO Classification of Tumors of Soft Tissue and Bone. Fletcher CDM, Bridge JA, Hogendoorn PCW and Mertens F (eds). IARC Press, Lyon, pp305-310, 2013.

13. Kim GY, Park JH, Kim YW, Jung WW, Unni KK and Park YK: Absence of amplification of HER-2/neu (c-erbB-2) gene in Ewing's sarcoma: a real-time polymerase chain reaction method. Pathol Res Pract 200: 663-667, 2004.

14. Farrand K, Jovanovic L, Delahunt B, et al: Loss of heterozygosity studies revisited: prior quantification of the amplifiable DNA content of archival samples improves efficiency and reliability. J Mol Diagn 4: 150-158, 2002.

15. Bibikova M and Fan JB: GoldenGate assay for DNA methylation profiling. Methods Mol Biol 507: 149-163, 2009.

16. Khoury JD: Ewing sarcoma family of tumors. Adv Anat Pathol 12: 212-220, 2005.

17. Fulda S, Küfer MU, Meyer E, van Valen F, Dockhorn-Dworniczak B and Debatin KM: Sensitization for death receptor- or drug-induced apoptosis by re-expression of caspase-8 through demethylation or gene transfer. Oncogene 20: 5865-5877, 2001 .

18. López-Guerrero JA, Pellín A, Noguera R, Carda C and Llombart-Bosch A: Molecular analysis of the 9p21 locus and p53 genes in Ewing family tumors. Lab Invest 81: 803-814, 2001.

19. Rathi A, Virmani AK, Harada K, et al: Aberrant hypermethylation of the HIC1 promoter is a frequent event in specific pediatric neoplasms. Clin Cancer Res 9: 3674-3678, 2003.

20. Martín-Subero JI, Kreuz M, Bibikova M, et al; Molecular Mechanisms in Malignant Lymphomas Network Project of the Deutsche Krebshilfe: New insights into the biology and origin of mature aggressive B-cell lymphomas by combined epigenomic, genomic, and transcriptional profiling. Blood 113: 2488-2497, 2009.

21. Serao NV, Delfino KR, Southey BR, Beever JE and Rodriguez-Zas SL: Cell cycle and aging, morphogenesis, and response to stimuli genes are individualized biomarkers of glioblastoma progression and survival. BMC Med Genomics 4: 49, 2011.

22. Giovannini M, Selleri L, Hermanson GG and Evans GA: Localization of the human oncostatin M gene (OSM) to chromosome 22q12, distal to the Ewing's sarcoma breakpoint. Cytogenet Cell Genet 62: 32-34, 1993.

23. Weiss TW, Simak R, Kaun C, et al: Oncostatin M and IL-6 induce u-PA and VEGF in prostate cancer cells and correlate in vivo. Anticancer Res 31: 3273-3278, 2011.

24. Ignatov A, Bischoff J, Ignatov T, et al: APC promoter hypermethylation is an early event in endometrial tumorigenesis. Cancer Sci 101: 321-327, 2010.

25. Shu Y, Wang B, Wang J, Wang JM and Zou SQ: Identification of methylation profile of HOX genes in extrahepatic cholangiocarcinoma. World J Gastroenterol 17: 3407-3419, 2011.

26. Fiegl H, Windbichler G, Mueller-Holzner E, et al: HOXA11 DNA methylation - a novel prognostic biomarker in ovarian cancer. Int J Cancer 123: 725-729, 2008.

27. Avigad S, Shukla S, Naumov I, et al: Aberrant methylation and reduced expression of RASSF1A in Ewing sarcoma. Pediatr Blood Cancer 53: 1023-1028, 2009.

28. Harada K, Toyooka S, Maitra A, et al: Aberrant promoter hypermethylation and silencing of the RASSF1A gene in pediatric tumors and cell lines. Oncogene 21: 4345-4349, 2002.

29. Garcia-Manero G: Demethylating agents in myeloid malignancies. Curr Opin Oncol 20: 705-710, 2008. 